Thipphaya Cherdhirankorn Wanchai Lerdwijitjarud Anuvat Sirivat Ronald G. Larson

\section{Dynamics of vorticity stretching and breakup of isolated viscoelastic droplets in an immiscible viscoelastic matrix}

blend of relatively lower Wi number of 0.21 , one cycle of oscillation in Def* was observed before reaching steady state negative values when $C a$ number was varied between 3 and 14. The steady state Def* varies inversely with $C a$ number, with a stronger dependence for the blend with higher Wi number. The magnitude of oscillation increases with increasing $\mathrm{Ca}$ and Wi numbers. The critical $C a$ was found to be 12 and 14 for the two blends studied; these values are about 30 times greater than that of Newtonian blends.

Keywords Viscoelastic droplet . Vorticity stretching $\cdot$ Immiscible blends

\section{Introduction}

For Newtonian polymer blends in a simple shear flow, the morphology of the minor (or droplet) phase is governed mainly by two dimensionless parameters: the viscosity ratio, i.e., the ratio of droplet viscosity to matrix viscosity; and the capillary number, the ratio of viscous to interfacial forces (Taylor 1932, 1934):

$C a=\frac{D_{o} \dot{\gamma} \eta_{m}}{2 \Gamma}$

where $\dot{\gamma} \eta_{m}$ is the viscous shear stress, with $\dot{\gamma}$ the shear rate and $\eta_{m}$ the matrix viscosity, $\mathrm{D}_{\mathrm{o}}$ is initial droplet diameter, and $\Gamma$ is the interfacial tension.

A steady applied shearing flow that is not so fast as to lead to droplet rupture will eventually stretch a droplet into a roughly ellipsoidal steady-state shape, and the steady-state degree of deformation, defined as the deformation parameter Def, is approximately linearly related to the capillary number as (Taylor 1932, 1934):

Def $\equiv \frac{a-b}{a+b}=C a \frac{19 \eta_{r}+16}{16 \eta_{r}+16}$

where $\mathrm{a}$ and $\mathrm{b}$ are the lengths of the major and minor axis of the deformed droplet, respectively. Taylor (1934) predicted that the critical point at which the viscous force overcomes the interfacial force leading to droplet breakup occurs at $\mathrm{Ca}_{\mathrm{c}} \approx 0.5$ and $\mathrm{Def}_{\mathrm{c}} \approx 0.5$ for a steady (or quasi-steady, if the flow rate is very slowly increased) simple shearing flow with a viscosity ratio of around unity. Here, the subscript "c" stands for the critical condition for breakup. These basic predictions have 
been confirmed and refined in a number of detailed single-droplet experiments (Rumscheid and Mason 1961; Grace 1982; Bentley and Leal 1986; Guido and Villone 1998). However, when the viscosity ratio is higher than four, no breakup can be observed (Grace 1982). These results show that for Newtonian fluids, droplet deformation and breakup is strongly influenced by viscosity ratio, a result that emphasizes the importance of controlling this parameter carefully in any attempt to study the effects of other factors, such as viscoelasticity, on droplet deformation and breakup.

The above results were obtained for Newtonian droplets and matrix fluids; however, most polymers are viscoelastic under normal processing conditions, and so elasticity of the droplet and matrix phases should be an important factor affecting the behavior of droplets under a flow field. It has long been noticed, for example, that in blends of viscoelastic polymer melts, the steady-state average droplet size that results from breakup and coalescence of droplets under shear corresponds to a much higher capillary number than is seen in blends of Newtonian liquids at comparable viscosity ratios. Wu (1987) observed, for example, that the steady-state droplet size in extruded viscoelastic polymer blends at a viscosity ratio of unity is around ten times higher than would be obtained for Newtonian components at the same viscosity and at the same shear rate. Lerdwijitjarud et al. (2002) found that in blends of $20 \%$ polystyrene in polyethylene sheared in a rheometer, the steady-state droplet size corresponded to a capillary number ranging from 2 to 30, depending on the relative magnitudes of the normal stress differences in the droplet and matrix phases and on the viscosity ratio. These capillary numbers are from 4 to 80 times higher than the critical capillary number for breakup of a Newtonian droplet in a Newtonian matrix. While it might be thought that this large increase in droplet size and hence capillary number could be due in part to coalescence effects present in blends, Lerdwijitjarud et al. (2003) showed recently that in a $20 \%$ blend of a Newtonian liquid in a Newtonian matrix the steady-state capillary number is actually lower than the critical capillary number for breakup of an isolated droplet, i.e., the disturbances to the flow produced by the presence of the other droplets enhances breakup of a given droplet to an extent that more than offsets any increase in droplet size due to coalescence. Thus, the high steady-state capillary numbers observed in blends of viscoelastic polymers must be attributed to the role of viscoelasticity.

Elasticity in the droplet or matrix phase can be quantified by the Weissenberg number $\mathrm{Wi}$, a ratio of elastic to viscous forces, which we here will estimate using either the first normal stress difference $\mathrm{N}_{1}$ or elastic modulus $\mathrm{G}^{\prime}$ as a measure of the elastic forces and the shear stress as a measure of the viscous forces. Like the capillary number, the Weissenberg number increases with increasing shear rate, since elastic forces generally increase with shear rate more rapidly than do viscous forces. For a given droplet size, Wi and $\mathrm{Ca}$ are qualitatively proportional to each other; however, since $\mathrm{Ca}$ depends on droplet diameter and Wi does not, these two dimensionless numbers can be varied independently by varying both the shear rate and the droplet size for a given pair of viscoelastic fluids. Since both phases can be elastic, there are two Weissenberg numbers, the droplet Weissenberg number $\mathrm{Wi}_{\mathrm{d}}$ and the matrix Weissenberg number $\mathrm{Wi}_{\mathrm{m}}$. Since the elastic stresses in the droplet depend on the strength of the flow in the droplet, which is, in turn dependent on the viscosity ratio (more viscous droplets have weaker internal flows), it is clear that in general there is a coupling between the viscosity ratio and the strength of the elastic forces in the droplet. To minimize this influence, in what follows, we will work with fluids having viscosity ratios near unity, and use the macroscopic shear rate to characterize the flow inside the droplet. That is, following the work of Lerdwijitjarud et al. (2002), we will not attempt to use the actual flow in the droplet to estimate the droplet Weissenberg number, but will characterize the elasticity of the droplet using $\mathrm{Wi}_{\mathrm{d}}$ defined by the droplet fluid properties at the macroscopic shear rate measured on the pure droplet fluid in a rheometer.

Recently, single viscoelastic droplets in Newtonian or viscoelastic matrices have been observed microscopically in simple shearing flows. Lerdwijitjarud et al. (2003) observed deformation and breakup of isolated droplets of weakly elastic fluid $\left(\mathrm{Wi}_{\mathrm{d}} \leq 0.02\right)$ in a Newtonian matrix, and found that droplet elasticity produces a slight (up to around 20\%) increase in $\mathrm{Ca}_{\mathrm{c}}$, the critical capillary number for droplet breakup. The breakup mechanism appeared to be similar to that in a Newtonian fluid; i.e., the droplet deformed increasingly in the flow direction as the shear rate was gradually increased, until breakup occurred. Elasticity of the droplet produced a reduction in the degree of deformation at any given shear rate and a greater critical deformation at breakup, resulting in a higher $\mathrm{Ca}_{\mathrm{c}}$. However, at the highest Weissenberg number, this effect appeared to be saturating, leading to only a modest increase in $\mathrm{Ca}_{\mathrm{c}}$.

Mighri et al. (1998) investigated the influence of elastic properties on droplet deformation and on the critical shear rate, or critical capillary number $\mathrm{Ca}_{c}$ for breakup. The deformed elastic droplet was roughly spheroidal with slightly sharpened edges while a Newtonian droplet retained smooth curved ends. Mighri et al. (1998) reported that the degree of droplet deformation, the critical shear rate for breakup, and the breakup time after startup of shearing increased with increasing elasticity ratio between the droplet and the matrix fluids. They defined the elasticity ratio, $\mathrm{k}^{\prime}$, as the ratio of the Maxwell relaxation time $(\lambda)$ of the droplet phase to that of the matrix phase, where $\lambda=N_{1 \mathrm{i}} / 2 \dot{\gamma}^{2}$, 
where $\mathrm{N}_{1 \mathrm{i}}$ is the first normal stress difference. Breakup occurred by an unsteady deformation of the droplet into a thin and long thread, followed by end pinching, and undulations along the droplet surface, finally resulting in a series of alternating large and small (or "satellite") droplets. Moreover, they found that for a low or modest elasticity ratio, $\mathrm{k}^{\prime} \leq 4$, the critical capillary number $\mathrm{Ca}_{\mathrm{c}}$ for droplet breakup in steady shearing flow increased with increasing $\mathrm{k}^{\prime}$, reaching $\mathrm{Ca}_{\mathrm{c}} \approx 1.75$ at high elasticity ratio, $\mathrm{k}^{\prime} \approx 4$, compared to $\mathrm{Ca}_{\mathrm{c}} \approx 0.5$ for Newtonian droplets. Thus, droplet resistance to deformation and breakup increases with increasing elasticity ratio of droplet to matrix phase. While the elasticities of the droplets studied by Mighri et al. (1998) were higher than those studied by Lerwijitjarud et al. (2003), the droplets in both studies deformed in the flow direction, and the increase in critical capillary number produced by elasticity saturated at a relatively modest values near unity, far below the steady-state values observed in highly viscoelastic polymer blends. These studies, and those described below, suggest that large increases in $\mathrm{Ca}_{\mathrm{c}}$ result from a new mode of droplet deformation and breakup for highly elastic droplets.

A new, viscoelastic, mode of droplet deformation, that of droplet extension in the vorticity direction (perpendicular to both the shear and shear gradient directions) was apparently first reported for an elastic polymer droplet in a polymer matrix by Bartram et al. (1975). An apparently related phenomenon of widening of an extended viscoelastic droplet in a highly viscoelastic matrix was later observed by Levitt et al. (1996), who suggested that there is a relationship between the second normal stress difference of droplet and matrix phase and the degree of widening. Hobbie and Migler (1999) studied dilute emulsions of viscoelastic droplets in viscoelastic matrices at high shear rate and also observed elongation of the droplet in the vorticity direction at $\dot{\gamma} \sim 280 \mathrm{~s}^{-1}$ for viscosity ratio $\eta_{\mathrm{r}}=1.8$. By extrapolating data at high shear rates to lower rates, they obtained critical capillary numbers for droplet vorticity alignment of around 53, 13, and 11 for viscosity ratios, $\eta_{\mathrm{r}}$, of 1.8 , 22 , and 240 , respectively. The increase in critical capillary number for vorticity alignment with viscosity ratio is consistent with a mechanism involving droplet elasticity, since a higher droplet viscosity would require a higher external flow rate to attain the same internal flow rate within the droplet, which would be needed to maintain a high elasticity of the droplet fluid.

Migler (2000) observed the deformation of highly elastic droplets in a polymeric matrix under a shear flow. The viscosity ratio was near unity, but the elasticity ratio of the droplet to the matrix was higher than 100; that is, the matrix phase was nearly Newtonian under the conditions of the experiments. In a weak shear and for small droplets $[\mathrm{Ca}<5]$, the droplet orientation was found to be along the flow direction, whereas in a strong shear and for large droplets $[\mathrm{Ca}>5]$, the orientation was along the vorticity axis with a broad distribution of aspect ratios.

Mighri and Huneault (2001) studied the deformation and breakup of a single droplet of viscoelastic Boger fluid in a Newtonian matrix, sheared in a transparent Couette flow cell. At low shear rate, they found that the steady-state deformation increased with shear rate as expected, but above a critical shear rate [Ca 5$]$ the deformed drop began contracting in the flow direction and changed its orientation to the vorticity axis. With further increases in shear rate, this elongation in the vorticity direction increased until breakup finally occurred at a capillary number no higher than $\mathrm{Ca} \sim 35$. They proposed that the critical shear stress for reorientation of the droplet in the vorticity direction was probably related to the values of the first and second normal stress differences and their dependencies on shear rate. They also suggested that this reorientation occurred because of a the flow-induced circulatory flow in the droplet that produced an elastic circular hoop stress in the plane containing the shear and the shear gradient directions that squeezed fluid out along the axis perpendicular to this plane, that is, along the vorticity axis (Hobbie and Migler 1999; Migler 2000). They surmised that in a startup of a steady shearing flow, the deforming viscous stress rapidly reached steady state, but the normal stresses generated by the dispersed phase required a longer time, which caused a gradual increase in droplet elongation along vorticity axis until either a steady-state deformation was reached or breakup occurred. When a droplet was highly stretched in the vorticity direction, they observed small rocking instabilities in the velocity gradient direction causing the two ends of the droplet to sample significantly different velocities periodically, which ultimately tore apart the droplet into two or more smaller drops.

In our work reported below, we take a further step towards understanding the behavior of commercial blends by using elastic polymer melts for both the droplet phase and the matrix phase. To neutralize any effect of variations in the viscosity ratio, we choose pairs of commercial polymers whose viscosity ratios remain relatively constant near unity when shear rate is varied. Using a flow cell mounted on an optical microscope, we observe the transient deformations of isolated droplets after startup of steady shear flow between parallel disks. By varying droplet size and shear rate, the effects of capillary number and elasticity (or droplet Weissenberg number) are isolated and investigated at fixed viscosity ratio very near unity. Our findings confirm those of Mighri and Huneault, who used nearly Newtonian matrix fluids, but, in addition, we find that when both the droplet fluid and the matrix fluid are highly elastic, droplet deformations during start-up of steady shearing show large oscillations before reaching steady state. 


\section{Experiments}

\section{Materials}

High density polyethylene and polystyrene (suppliers and grades given in Table 1) were used as matrix and droplet phases, respectively. Both polystyrene grades were obtained from the manufacturers in the form of flake; they were crushed and size-selected by passing the flakes through a $425-\mathrm{mm}$ sieve. To eliminate volatile components, all polymers were heated to around $80^{\circ} \mathrm{C}$ under vacuum for $12 \mathrm{~h}$. The polymer blend systems and their experimental temperatures are listed in Table 2.

\section{Rheological characterization}

Each polymer was molded into a disk, $25 \mathrm{~mm}$ in diameter and $1 \mathrm{~mm}$ thick by using a compression mold (Wabash, model V50H18-CX) at $145^{\circ} \mathrm{C}$ for HDPE1 and PS1, and at $135^{\circ} \mathrm{C}$ for HDPE2 and PS2 under a force of 10 tons. We used a cone-and-plate rheometer (Rheometrics Scientific: Model ARES, 25-mm plate diameter with cone angle $0.1 \mathrm{rad}$ ) to obtain viscosities and first normal stress differences of the pure polymers. From the rheological properties of pure polymers at various temperatures, the desired pairs of polymers and operating temperatures were selected for further study; and their steady-state viscosities and first normal stress differences as functions of shear rate are shown in Fig. 1a,b. At low shear rates, the ratio of droplet-to-matrix first normal stress differences $\mathrm{N}_{1 \mathrm{r}}$ of system $\mathrm{B}$ could not be precisely determined due to the force-measurement limitations of the rheometer. However, in the low-shear-rate and low-frequency regimes, $N_{1}(\dot{\gamma})$ is approximately equal to twice $\mathrm{G}^{\prime}(\omega)$ at $\dot{\gamma}=\omega$, thus $\mathrm{N}_{1 \mathrm{r}}$ can be estimated by the corresponding ratio of storage moduli, $\mathrm{G}_{\mathrm{r}}^{\prime}$. The Weissenberg number (Wi) of both matrix and dispersed phases at testing conditions is also given in Table 3 . The viscosity ratios and $\mathrm{G}^{\prime}$ ratios of the two polymer systems are shown in Fig. 2. In addition, the stabilities of all polymers to thermal degradation were tested at their experimental temperatures by measuring the viscosity at a constant shear rate, $0.5 \mathrm{~s}^{-1}$, for $4 \mathrm{~h}$; in all cases the viscosity values remain unchanged, allowing us to conduct blend experiments on each system for periods as long as $4 \mathrm{~h}$.

\section{Observations of an isolated droplet in shearing flow}

Shearing apparatus To observe the droplet behaviors in simple shearing flow, we used a flow cell (Linkam CSS 450, Linkam Scientific Instruments Ltd., UK) consisting of two transparent quartz parallel disks mounted on an optical microscope (Leica DMRPX, Leica Imaging Systems LTd., Cambridge, England), and connected to a CCD camera (Cohu 4910, Cohu Inc., CA). In addition, the images were analyzed on a computer using the Scion image software.

Sample preparation HDPE used as the matrix polymer was molded into a disk $25 \mathrm{~mm}$ in diameter and $0.5-1 \mathrm{~mm}$ thick by compression molding at $145^{\circ} \mathrm{C}$ for HDPE1 and $135^{\circ} \mathrm{C}$ for HDPE2. Various PS droplets were introduced into the matrix by using a pin to put a small amount of PS powder on a HDPE disk, and then covering this with another HDPE disk to form a sandwich. The sample was then placed between the top and the bottom disks of the flow cell, both of which were brought into contact with the sample, which was then heated to the testing temperature.

Droplet shape relaxation time We attempted to determine the interfacial tensions by measuring the deformation parameter Def (cf. Eq. 2) of a retracting droplet vs time, which is known to decay exponentially (Luciania et al. 1997; Yamane et al. 1998):

$D e f=D e f_{o} \exp \left(-\frac{t}{\tau}\right)$

so that the slope of Def vs $t$ on a semi-log plot can be related the characteristic relaxation time for a single drop, $\tau$. By equating this characteristic relaxation time to that predicted by the Palierne model (Eq. 4) (Palierne 1990; Graebling et al. 1993), the apparent interfacial tension was then calculated from the following relation:

$\tau=\frac{\left(3+2 \eta_{r}\right)\left(16+19 \eta_{r}\right) r_{o} \eta_{m, o}}{40\left(1+\eta_{r}\right) \Gamma}$

To obtain images of the relaxing droplet after a large strain, the desired strain was imposed onto a selected drop in the field of view of the microscope which moved the droplet out of the field of view, then the droplet was left to relax for at least 40-50 min (which is equal to or greater than the droplet relaxation time) to ensure that the drop had returned to the spherical shape, and then the droplet was moved back into the field of view by imposing the same strain in the reverse direction. A hundred to 200 images were then recorded (10-20 s per frame) while the droplet relaxed its shape. For both systems, the droplet size was varied from around $100 \mu \mathrm{m}$ up to $400 \mu \mathrm{m}$ or thereabouts. For system A, with initial drop size of $96 \mu \mathrm{m}$, shear rate $0.21 / \mathrm{s}, \eta_{\mathrm{r}}$ equal to 0.79 , and $\eta_{\mathrm{r}}$ equal to $3150 \mathrm{Pas}$, the relaxation time of Eq. (3) was $362 \mathrm{~s}$ and the corresponding $\Gamma$ was $1.66 \mathrm{mN} / \mathrm{m}$. Figure 3a,b shows that the apparent interfacial tension values inferred from Eq. (4) increases with the droplet size. This dependence of apparent interfacial tension on droplet size is likely caused by the contribution of droplet elasticity to the relaxation of the droplet shape. For large enough droplets, relaxation should become slow enough that viscoelastic stresses relax too quickly to influence droplet shape relaxation and hence the rate of relaxation is governed by interfacial tension alone. Thus, the interfacial tension value obtained for large droplets is expected to be the most accurate. Unfortunately, because of the limitation in the ratio of a gap width to an initial droplet size, which was kept at greater than 5 , we cannot attain a regime in which the apparent interfacial tension becomes independent of droplet size; see Fig. 3. Therefore, the values of the interfacial tension for the polymer blend systems used in this work were taken from the literature (Brandrup and Immergut 1989), which are $5.79 \mathrm{mN} / \mathrm{m}$ for system A at $147^{\circ} \mathrm{C}$, and $5.92 \mathrm{mN} / \mathrm{m}$ for system $\mathrm{B}$ at $139^{\circ} \mathrm{C}$.

From the optical microscope, the droplet images were captured only from the top view, i.e., a view containing the flow and vorticity directions. Since only a projection of the droplet onto the vorticity plane can be imaged from this view, this view cannot determine the true lengths of the principal axes, because two of them (those in the flow and the shear-gradient directions) are not parallel to the vorticity plane. However, the lengths of these axes can be determined by using the affine angle of rotation of the droplet in the plane containing the flow and shear-gradient directions (Larson
Table 1 Properties of polymer blend components

${ }^{\mathrm{a}}$ Quoted by the manufacturers

\begin{tabular}{lllll}
\hline Polymer & Suppliers & Grade & $\mathrm{M}_{\mathrm{w}}{ }^{\mathrm{a}}$ & Melt Flow Index $(\mathrm{g} / 10 \mathrm{~min})$ \\
\hline PS1 & Polyscience & Cat\#18544 & 50,000 & - \\
PS2 & Polyscience & Cat\#23637 & $800-5,000$ & - \\
HDPE1 & Bangkok Polyethylene & $1600 \mathrm{~J}$ & - & 14 \\
HDPE2 & Aldrich & Cat\#42,801-9 & - & 42 \\
\hline
\end{tabular}


Table 2 Polymer blend systems

\begin{tabular}{lllc}
\hline Blend system & $\begin{array}{l}\text { Blend components } \\
\text { (drop/matrix) }\end{array}$ & Temperature $\left({ }^{\circ} \mathrm{C}\right)$ & $\begin{array}{l}\Gamma \\
(\mathrm{mN} / \mathrm{m})\end{array}$ \\
\hline $\mathrm{A}$ & PS1/HDPE1 & 147 & 5.79 \\
$\mathrm{~B}$ & PS2/HDPE2 & 139 & 5.92 \\
\hline
\end{tabular}
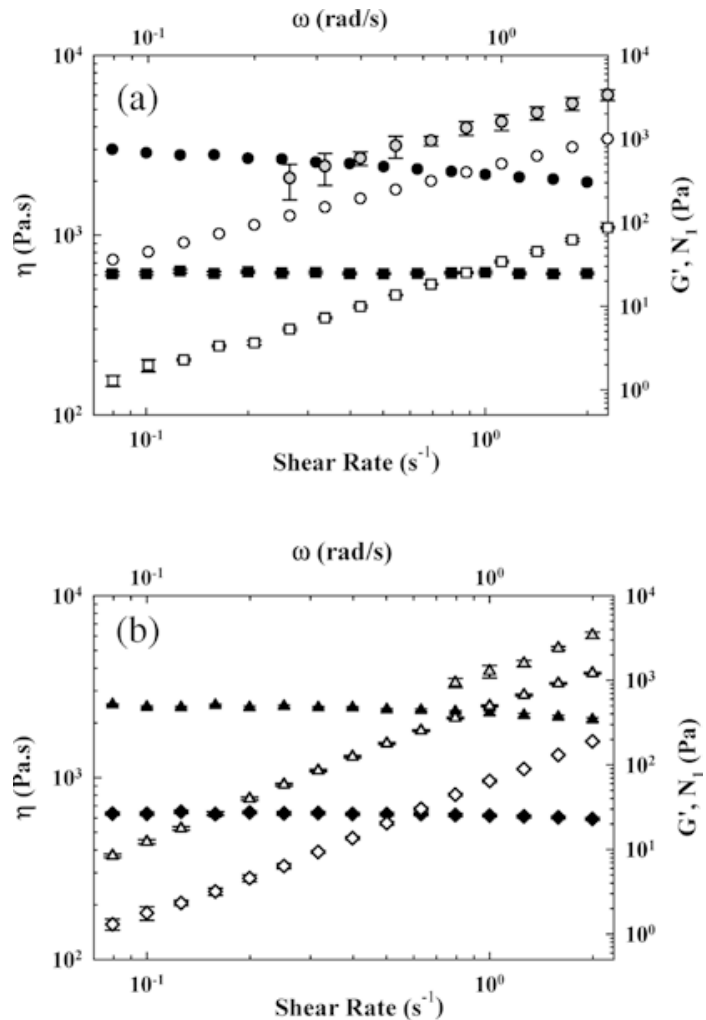

Fig. 1a,b Viscosity $\eta$, storage modulus $\mathrm{G}^{\prime}$, and first normal stress difference $N_{1}$ as functions of shear rate and frequency for each pure polymer at the temperatures at which the blend experiments were carried out: a matrix phase HDPE1 at $147^{\circ} \mathrm{C}, \eta$ (filled circles), $\mathrm{G}^{\prime}$ (open circles), and $\mathrm{N}_{1}$ (shaded circles), HDPE2 at $139^{\circ} \mathrm{C}, \eta$ (filled squares) and $\mathrm{G}^{\prime}$ (open squares); b droplet phase PS1 at $147^{\circ} \mathrm{C}, \eta$ (filled triangles), $\mathrm{G}^{\prime}$ (open triangles), and $\mathrm{N}_{1}$ (shaded triangles), $\mathrm{PS} 2$ at $139{ }^{\circ} \mathrm{C}: \eta$ (filled diamonds) and $\mathrm{G}^{\prime}$ (open diamonds)

1999) together with the condition of volume preservation, $\mathrm{D}_{\mathrm{o}}{ }^{3}=a b c$ (Almusallam et al. 2000).

Although the lengths of the principal axes can be approximated by using the method mentioned above, for convenience we use the lengths of the observable axes, shown in Fig. 4, to describe the behavior of each droplet. Thus, we define a modified deformation parameter Def* as

$D e f * \equiv \frac{a *-c}{a *+c}$

where the asterisk denotes that the deformation parameter is an apparent one obtained from the droplet image projected onto the flow-vorticity plane; see Fig. 4.

Transient deformation The deformed shapes were observed as a function of time from initial to steady-state shapes. Because the Linkham device has one stationary and one moving plate, a single droplet cannot be viewed continuously from startup of shearing to attainment of steady-state shape, since this droplet will pass out of the viewing plane after imposition of around ten strain units. However, since the behavior of a given isolated droplet is highly reproducible, the strain dependence of the deformation can be determined by combining the results of several experiments. In experiments of type 1, we first move the droplet out of the viewing window by imposing a shearing strain of less than 40 strain units. After allowing the droplet to relax its shape for at least $40 \mathrm{~min}$, an observed shear rate was imposed at the same strain but in opposite direction, eventually bringing the droplet back into the viewing window, where it can be observed during deformation. However, the droplet could not be subjected to a large strain in this type of experiment, since this would move the droplet again out of the viewing window. So, to obtain droplet deformation at large strains, we performed experiments of type 2 , in which we sheared continuously, and imaged the droplet each time it passed through the viewing window in its orbit around the axis of the rotation of the rotating plate. Typically, one orbit would require around 40 strain units. To get a clear image of the droplet without a high-speed camera, we stopped the flow briefly each time the droplet reached the center of the viewing image and video recorded its shape over a period of around $1 \mathrm{~s}$, which is a time much too short for the droplet to relax its shape significantly. Then the flow was resumed again until the droplet again reached the viewing window. By repeating the experiment on droplets of similar size, which were stopped at different times, we could assemble a consistent curve from multiple droplets of the droplet deformation versus time at a given shear rate. In the following, the results from different droplets will be presented using different symbols, showing the consistency of results combined from multiple droplets. In each experiment, the time for one revolution of a droplet was recorded with a stopwatch along with the time shown on the Linksys program. To avoid interactions with the plates, the ratio of the gap width to the initial diameter of a selected drop was kept higher than 10, and only droplets near the center of the gap were observed. The experiment was repeated with more than ten droplets with initial diameters around $75 \mu \mathrm{m}( \pm 10 \%)$ were used, and the imposed shear rate was $0.5 \mathrm{~s}^{-1}$. Similar experiments (only type 2 ) were carried out using other droplet sizes, 52,110, and $120 \mu \mathrm{m}$, but the same shear rate of $0.5 \mathrm{~s}^{-1}$. To separate the effect of shear rate from that of elasticity, another set of experiments were carried out on droplets of different sizes $[135,75$, and $46 \mu \mathrm{m}]$ but in which the shear rate varied
Table 3 The rheological data of blend systems

\section{- = Not measurable \\ ${ }^{a} \eta$ unit is Pa.s \\ ${ }^{\mathrm{b}} \mathrm{N}_{1}, \mathrm{G}$ ' unit is $\mathrm{Pa}$}

${ }^{\mathrm{c}}$ The Weisenberg number was calculated from the relation $\mathrm{Wi}=\left(2 \mathrm{G}^{\prime}\right) /[\eta(\dot{\gamma}) \cdot \dot{\gamma}]$

\begin{tabular}{|c|c|c|c|c|c|c|c|c|c|}
\hline \multirow[t]{2}{*}{ Blend system } & \multirow[t]{2}{*}{ Shear rate $\left(\mathrm{s}^{-1}\right)$} & \multicolumn{4}{|c|}{ Matrix } & \multicolumn{4}{|c|}{ Droplet } \\
\hline & & $\eta^{\mathrm{a}}$ & $\mathrm{N}_{1}{ }^{\mathrm{b}}$ & $\mathrm{G}^{\prime}$ & $\mathrm{W}_{\mathrm{i}}^{\mathrm{c}}$ & $\eta$ & $\mathrm{N}_{1}$ & $\mathrm{G}^{\prime}$ & $\mathrm{W}_{\mathrm{i}}^{\mathrm{c}}$ \\
\hline \multirow[t]{4}{*}{ A } & 0.28 & 2524 & 403 & 135 & 0.38 & 2426 & - & 70 & 0.21 \\
\hline & 0.3 & 2505 & 435 & 145 & 0.39 & 2425 & - & 79 & 0.22 \\
\hline & 0.5 & 2340 & 772 & 247 & 0.42 & 2340 & - & 179 & 0.31 \\
\hline & 0.8 & 2175 & 1359 & 400 & 0.46 & 2272 & 936 & 359 & 0.40 \\
\hline B & 1.0 & 612 & - & 34.2 & 0.11 & 619 & - & 64.7 & 0.21 \\
\hline
\end{tabular}



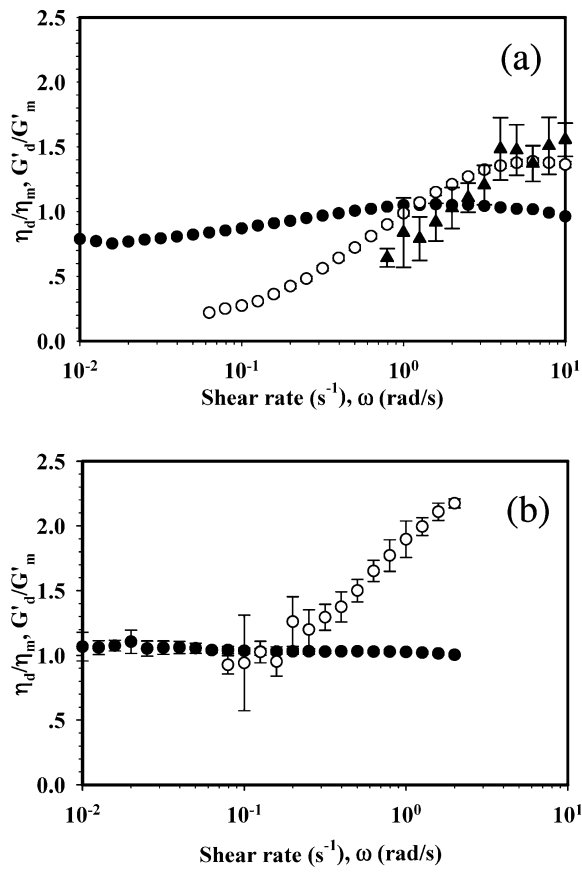

Fig. 2a,b Values of droplet to matrix ratios of viscosity $\eta$ (filled circles), storage modulus $\mathrm{G}^{\prime}$ (open circles), and first normal stress difference $\mathrm{N}_{1}$ (filled triangles) for: a system A PS1/HDPE1 at $147{ }^{\circ} \mathrm{C}$; b system B, PS2/HDPE2 at $139{ }^{\circ} \mathrm{C}$
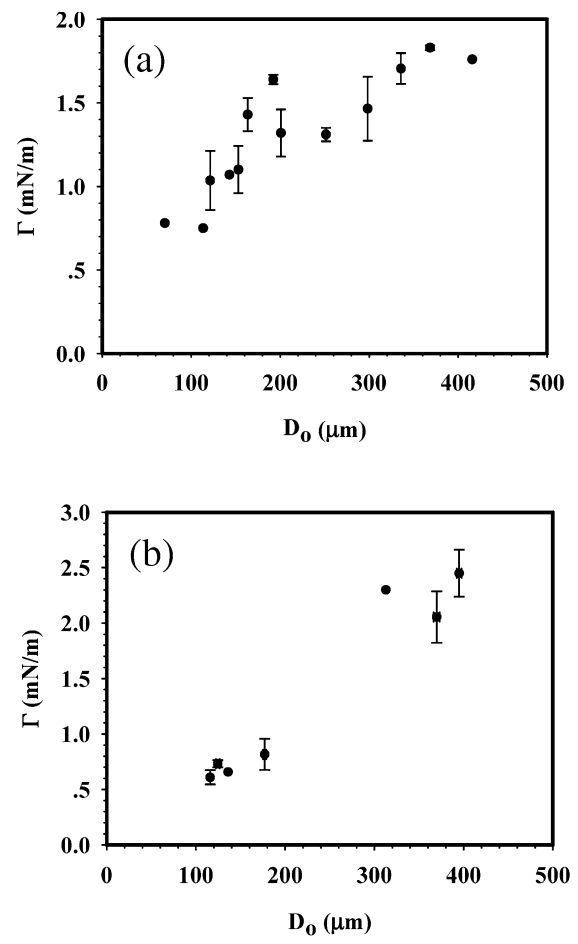

Fig. 3a,b Dependence of apparent interfacial tension value on droplet size for: a system A; b system B, as inferred from the Palierne formula, Eq. (4)

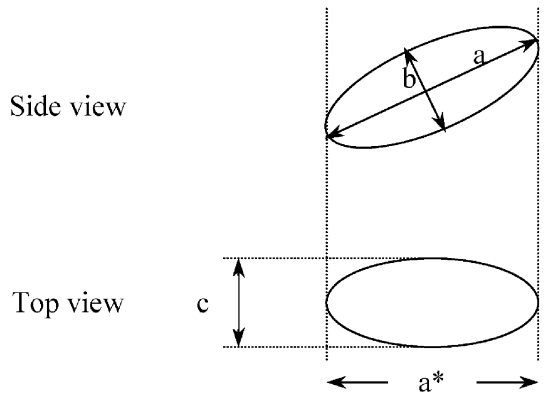

Flow direction

Fig. 4 Schematic drawing of a single drop observed from the "side" and "top" views by optical microscopy, a and b: the long and short axes of the droplet in the flow-gradient plane, $a^{*}:$ the a axis projected into the flow direction and $c$ : the principal axis in the radial direction

inversely with droplet size from 0.28 , to 0.5 , to $0.8 \mathrm{~s}^{-1}$. In this way we could vary the viscoelastic forces, which increase with increasing shear rate, while holding the capillary number fixed at around 8 by varying the droplet size inversely with the shear rate.

Steady-state deformation and breakup From the transient experiments, the required strain to reach steady state can be determined and was found to be around 2500 strain units. To determine the steady-state droplet shape as a function of capillary number, we carried out experiments up to high strains at two different shear rates and for different droplet sizes. The selected shear rates were 0.3 and $0.5 \mathrm{~s}^{-1}$ for system $\mathrm{A}$, and $1 \mathrm{~s}^{-1}$ for system $\mathrm{B}$, which are high enough that the needed strain could be achieved within an acceptable time, but not so high that the steady-state droplet shapes are too small to be imaged clearly. In addition to using these two shear rates, different droplet sizes were chosen to vary the capillary number at fixed elasticity. After loading a sample, droplets were allowed to relax to spherical shapes for a period of at least $50 \mathrm{~min}$, a bit longer than for the transient experiments described earlier, since some of the droplets used were larger and so needed somewhat longer to relax. A constant shear rate was then applied until a strain exceeding 2500 strain units had accumulated. When a selected droplet passed through the viewing window, the motor was stopped for less than a second and a video movie (speed $25 \mathrm{fps}$ ) was then recorded, as described earlier in the description of the experiments measuring transient droplet shapes. To ensure that the steady state deformation had indeed been attained, we repeated imaging the same droplet for several more passes of the droplet through the viewing window over a period of 5 to $10 \mathrm{~min}$. In addition, after turning off the flow the droplet shape relaxation was recorded at a video recording speed of $10-20 \mathrm{~s}$ per frame for approximately $1 \mathrm{~h}$.

When the droplet size was varied at fixed shearing rate, we found a critical droplet size above which no steady state-shape was obtained. For these droplets, the unstable shape of the droplet was recorded with time, by imaging the droplet each time it passed through the viewing window (as in the experiments discussed earlier), until the droplet broke.

\section{Results and discussion}

Deformation in steady shear flow

\section{Observations of droplet deformation}

We first consider system $\mathrm{A}$, in which both matrix and droplet phases are highly elastic with a $G^{\prime}$ ratio of 0.7 at 
$\omega=0.5 \mathrm{rad} / \mathrm{s}$. A shear rate of $0.5 \mathrm{~s}^{-1}$ and a droplet size of $75 \mu \mathrm{m}( \pm 10 \%)$ were selected for study at a well-controlled temperature $\left(147^{\circ} \mathrm{C}\right)$. We observed that the shape of the deformed drop, Def*, under steady shear flow oscillates before attaining a steady state shape, as shown in Figs. 5 and 6. We divide the transient deformation into five regimes. In the first regime, $a^{*}$, the principle axis projected in the flow direction, shown in Fig. 5c, oscillates with a periodicity of around $10 \mathrm{~s}$, while $c$, the length of the principle axis in the vorticity direction, shown in Fig. 5d, does not change from its initial value until near the end of regime 1. From the absence of a variation in $c$, we infer that this oscillation may arise from a droplet tilting or rotation around the vorticity (z) axis as shown schematically in Fig. 7. Eventually, near the end of regime 1, while it is still tilting or rotating, the droplet begins to contract in the $\mathrm{z}-\theta$ plane, and the $c$ axis increases slowly. In the second regime, the drop gradually elongates in the vorticity direction, and after around $500 \mathrm{~s}$ (or 250 strain units) elongation in the vorticity direction reaches its maximum at the end of this regime. At the maximum $\left|\operatorname{Def}^{*}\right|$ between the regime 2 and 3 , as shown in Fig. 5a, the $c$ axis is much larger than its initial value so that $c / \mathrm{D}_{\mathrm{o}} \sim 1.35$, while the a axis returns to a length nearly equal to its initial value (Fig. 5c). From volume conservation, this implies that the droplet is flattened in the $\mathrm{z}$ direction, as shown in Fig. 8. However, the deformed drop is not stable at this point because in regime 3 , the droplet rapidly contracts in the vorticity direction; see Fig. 5d. While contracting in the vorticity direction and stretching occur along the flow direction, cusps emerge out of each side of the droplet along the vorticity axis, as can be seen in Fig. 6k,1. After 1000 strain units have been imposed, the droplet stretches in the vorticity direction again (regime 4) until a steadystate shape is attained at around 2000 strain units in the fifth regime, as shown in Fig. 5b.

These phenomena do not occur in system B, whose droplet and matrix phase elasticities are both lower than in system A, but whose elasticity ratio of droplet to matrix phase is higher than in system A, as shown in Table 3 and Figs. 1 and 2. When a constant shear rate of $0.4 \mathrm{~s}^{-1}$ is applied to system $\mathrm{B}$, the deformation parameter rises rapidly from zero to around 0.09 , and then slowly and roughly exponentially decreases with time until attaining a steady-state value shown in Fig. 9. This finding is similar to that of Mighri and Huneault (2001) who reported that under a strong shearing flow, an elastic droplet in a Newtonian matrix rapidly stretches along the flow direction, and then gradually contracts in this direction after the first normal stress difference in the droplet has developed sufficiently. Our Fig. 9 is very similar to Fig. 7a of Mighri and Huneault, except that in the latter, the droplet eventually becomes aligned in the vorticity rather than in the shear direction, i.e., the deformation parameter becomes negative. We also obtain negative steady-state values of Def* for system B at higher values of $\mathrm{Ca}$ (as will be presented below). The similarity between our results for system B in a torsional plate-plate flow and the results of Mighri and Huneault
Fig. 5 The time-dependent deformation of $75( \pm 10 \%) \mu \mathrm{m}$ drops after startup of steady shear at a rate, $0.5 \mathrm{~s}^{-1}$, for system A: a Def* vs time on a log time scale; b Def* vs time on a linear time scale; $\mathbf{c} a^{*} / \mathrm{D}_{\mathrm{o}}$ vs time on a $\log$ time scale, $\mathbf{d} c / \mathrm{D}_{\mathrm{o}}$ vs time on a log time scale. [initial droplet sizes $(\mu \mathrm{m}): 76$ (filled circles), 68 (dotted circles), 74 (inverted filled triangles), 69 (inverted open triangles), 71 (filled squares), 85 (dotted squares), 76 (open diamonds), 75 (dotted diamonds), 74 (filled triangles), 70 (dotted triangles), and 79 (filled circles)]
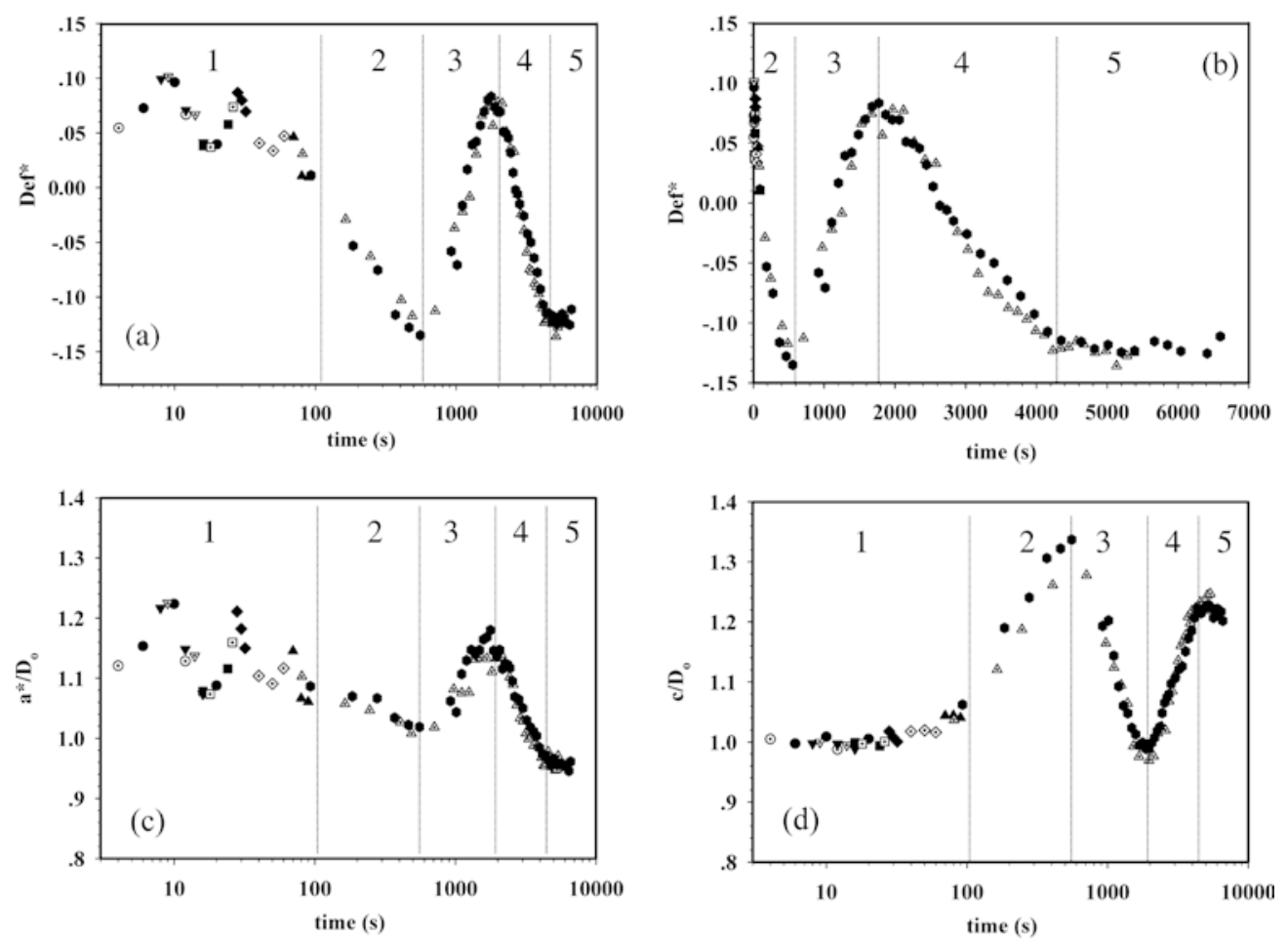


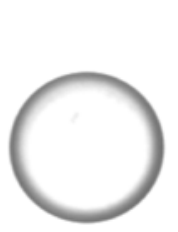

(a) $0 \mathrm{~s}$

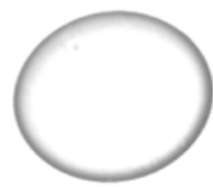

(d) $28 \mathrm{~s}$

$100 \mu \mathrm{m}$

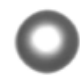

(g) $93 \mathrm{~s}$

(h) $185 \mathrm{~s}$

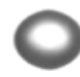

(1) $1773 \mathrm{~s}$

(m) $2350 \mathrm{~s}$

(p) 4350

Fig. 6a-p Sequence of images of deformed droplets of initial radius $75 \mu \mathrm{m}( \pm 10 \%)$ after startup of a steady shear at a rate of $0.5 \mathrm{~s}^{-1}$ for system A: a-f images of different droplets with lens magnification of 20x $\left[\mathrm{D}_{\mathrm{o}}=69,69,71,76,75\right.$, and $74 \mu \mathrm{m}$, respectively]; $\mathbf{g}-\mathbf{p}$ images of a single droplet with the lens magnification of $4 \mathrm{x}$ $\left[\mathrm{D}_{\mathrm{o}}=79 \mu \mathrm{m}\right]$
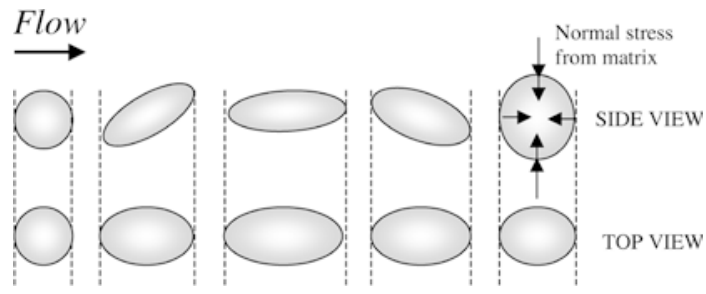

Fig. 7 Schematic drawing of droplet rotation in the flow-gradient plane

in a circular Couette flow implies that the unusual oscillatory droplet deformation we observe for system A is not an artifact of our system geometry. We speculate that the oscillations arise only in blends for which both phases are highly elastic, and occur because the different growth periods for the first normal stress differences of the two phases produce time-delayed imbalances in the normal stress conditions on the droplet surface.

\section{Effect of $\mathrm{Ca}$ on droplet deformation}

In this section, the elasticity of the droplet and matrix phases are held constant by holding the shear rate fixed at $0.5 \mathrm{~s}^{-1}$. At this shear rate, where the $\mathrm{G}^{\prime}$ ratio for blend

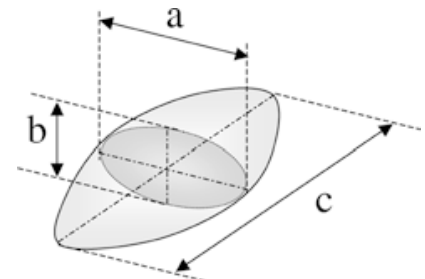

Fig. 8 Sketch of a steady-state deformed droplet

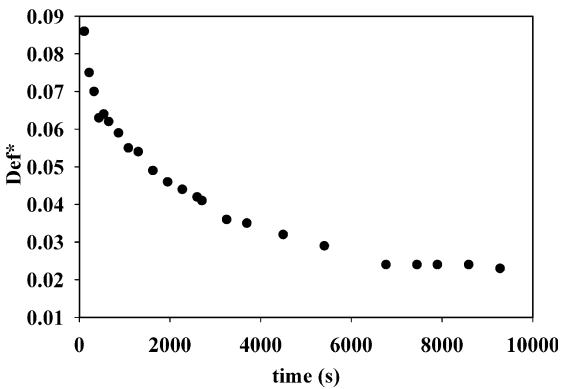

Fig. 9 Time-dependent deformation of a 204- $\mu \mathrm{m}$ droplet under constant shear rate, $0.4 \mathrm{~s}^{-1}$, for system $\mathrm{B}$

A at $\omega=0.5 \mathrm{rad} / \mathrm{s}$ is 0.7 , we selected various initial droplet sizes to vary the capillary number at a fixed droplet/matrix elasticity ratio. The deformation parameter Def* vs time from the second to fifth regimes of the 52, 79, 110, and $120 \mu \mathrm{m}$-drops is shown in Fig. 10a. It can be seen in Fig. 10b that between the second and the fourth regimes, droplets with higher $\mathrm{Ca}$ show a greater deformation in the flow direction (larger $a^{*} / \mathrm{D}_{\mathrm{o}}$ ). However, for all $\mathrm{Ca}$ values in these experiments, nearly the same value of $c / D_{o}$ is reached at its minimum point at the boundary between regimes 3 and 4 , as shown in Fig. 10c. For Ca values of 5 and 8, steady shapes are attained at strains around 1300 and 2000 (times of 2600 and $4000 \mathrm{~s}$ ), respectively, with larger droplet deformations (more negative Def*) occurring with increasing capillary number. The larger droplets with diameters of 110 and $120 \mu \mathrm{m}$ (higher capillary numbers of 11 and 12), do not attain steady-state shapes. For these droplets, the vorticity axis, $c$, rapidly increases for a long period and the axis in the flow direction $\left(a^{*}\right)$ slightly decreases until the drops eventually break (Fig. 10b,c).

\section{Effect of elasticity on droplet deformation}

To investigate the effect of elasticity in system $A$, the capillary number was kept constant at 8 and the shear rate and drop size were varied inversely with respect to each other. Shear rates of $0.28,0.5$, and $0.8 \mathrm{~s}^{-1}$ were chosen and the droplet sizes used for these shear rates were 135 (two runs), 70-79 (repeated experiments), and 

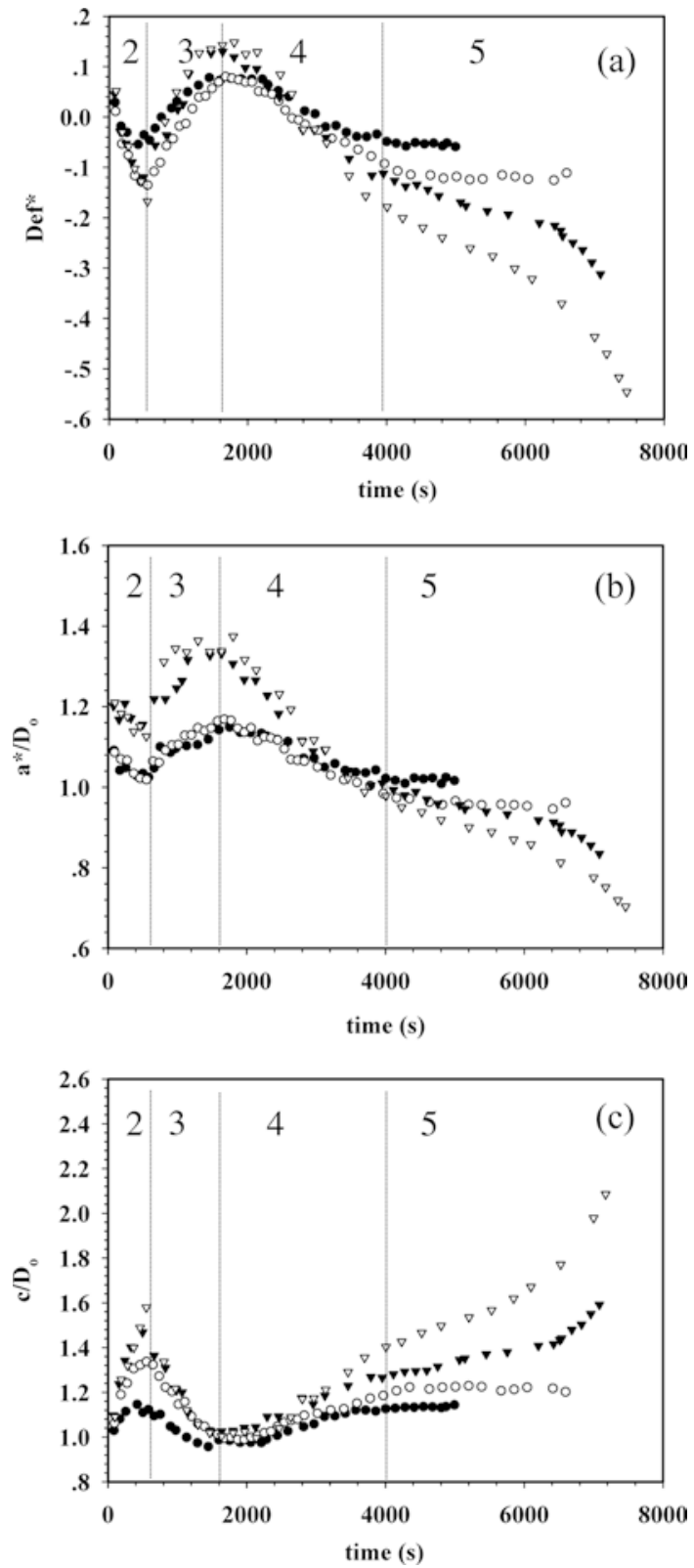

Fig. 10a-c Time-dependent droplet deformation at different values of $\mathrm{Ca}$, controlled by changing the droplet diameter $\mathrm{D}_{\mathrm{o}}$ $\left[\mathrm{D}_{\mathrm{o}}=52 \mu \mathrm{m} ; \mathrm{Ca}=5\right.$ (filled circles), $\mathrm{D}_{\mathrm{o}}=79 \mu \mathrm{m} ; \mathrm{Ca}=8$ (open circles), $\quad \mathrm{D}_{\mathrm{o}}=110 \mu \mathrm{m} ; \mathrm{Ca}=11$ (inverted filled triangles), $\mathrm{D}_{\mathrm{o}}=120 \mu \mathrm{m} ; \mathrm{Ca}=12$ (inverted open triangles)] at the same shear rate $0.5 \mathrm{~s}^{-1}$ (and therefore the same elasticity) for system A: a Def*; b $a^{*} / \mathrm{D}_{\mathrm{o}} ; \mathbf{c} c / \mathrm{D}_{\mathrm{o}}$

$45 \mu \mathrm{m}$, respectively. Figure 11a shows that there is a significant decrease in the maximum value of Def* vs $t$ (at a strain of around 900) as the shear rate (and hence the elasticity) increases. As shown in Figs. 11b,c, this decrease results mainly from a decrease in $a^{*} / \mathrm{D}_{\mathrm{o}}$, rather than an increase in $c / \mathrm{D}_{\mathrm{o}}$. The steady-state value of Def* in regime five is nearly the same for all shear rates.
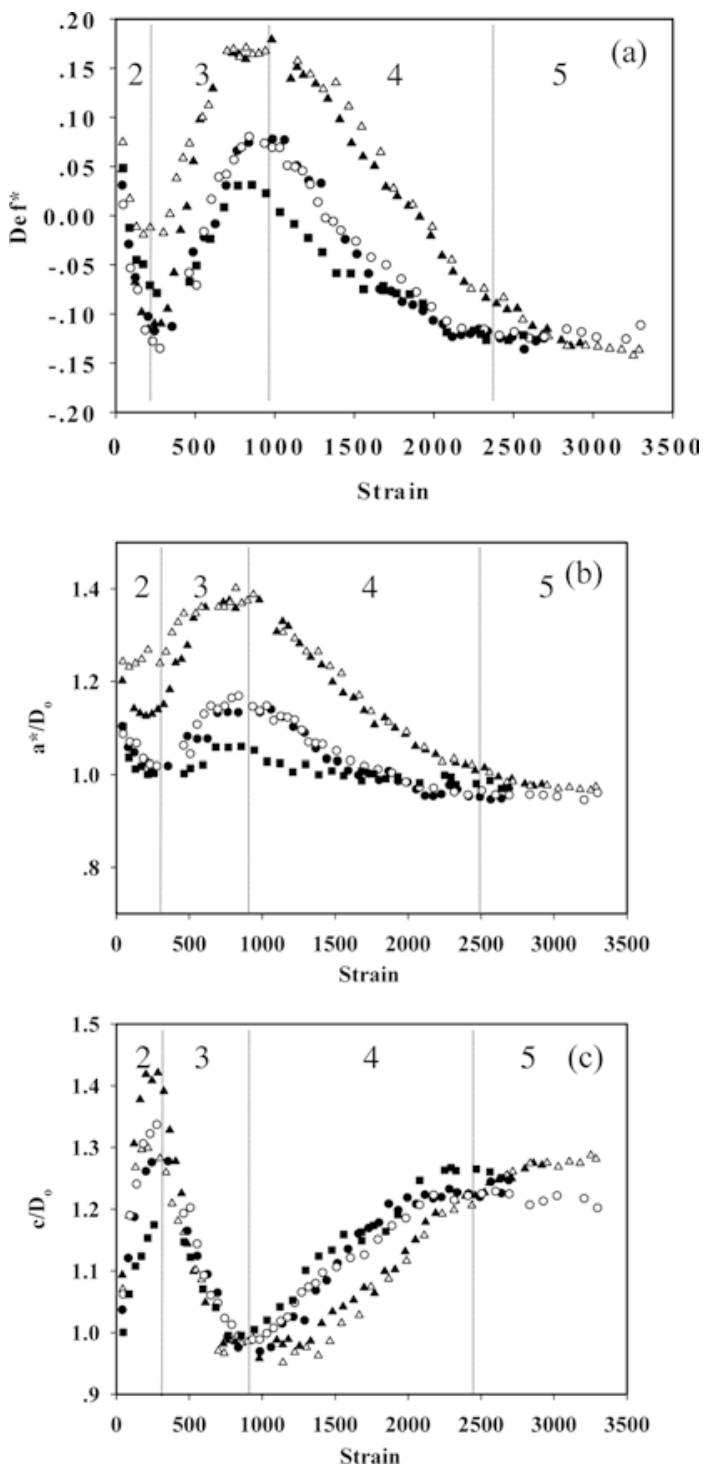

Fig. 11a-c Time-dependent droplet deformation at different shear rates (and therefore different elasticities), at a capillary number $\mathrm{Ca}$ of 8 , held fixed by varying the initial droplet diameter inversely with the shear rate for system A: a Def* vs time; $\mathbf{b} a^{*} / \mathrm{D}_{\mathrm{o}}$ vs time; $\mathbf{c} c / \mathrm{D}_{\mathrm{o}}$ vs time. Three different shear rates were used. (1): $\dot{\gamma}=0.28 \mathrm{~s}^{-1}$, with $\mathrm{D}_{\mathrm{o}}=135 \mu \mathrm{m}$ (filled triangles) and a repeat run at this shear rate with the same droplet size, $\mathrm{D}_{\mathrm{o}}=135 \mu \mathrm{m}$ (open triangles)]. (2): $\dot{\gamma}=0.5 \mathrm{~s}^{-1}: \mathrm{D}_{\mathrm{o}}=70 \mu \mathrm{m}$ (filled circles), and a repeat run with a slightly larger droplet, $\mathrm{D}_{\mathrm{o}}=79 \mu \mathrm{m}$ (open circles). $(3) \dot{\gamma}=0.8 \mathrm{~s}^{-1}$, $\mathrm{D}_{\mathrm{o}}=45 \mu \mathrm{m}$ (filled squares)]

\section{Steady-state deformation and droplet breakup mechanism}

The strains required to attain steady state at each shear rate were determined at shear rates of 0.3 and $0.5 \mathrm{~s}^{-1}$ for system $\mathrm{A}$, and at $1 \mathrm{~s}^{-1}$ for system $\mathrm{B}$. By increasing the droplet size, the capillary number was varied. As shown in Fig. 12, when $\mathrm{Ca}$ increases, the steady-state defor- 
mation in the vorticity direction increases; i.e., Def* becomes more negative. This contrasts with the behavior of a Newtonian system where the steady-state deformation in the flow direction increases monotonically with Ca (Taylor 1934; Tsakalos et al. 1998; Guido and Villone 1998). Figure 12 shows that a droplet in both systems starts to stretch in the vorticity direction at $\mathrm{Ca}$ around 3. Whereas Mighri and Huneault (2001), using less viscous liquids, found that at $\mathrm{Ca}$ less than 10 , an elastic droplet in a Newtonian matrix deforms along the flow direction with steady-state deformation increasing with increasing $\mathrm{Ca}$, until $\mathrm{Ca}$ reaches roughly 5 , above which the droplet starts contracting in the flow direction. At each value of $\mathrm{Ca}$ a droplet in our system $\mathrm{B}$ deforms less in the vorticity direction than one in system A (lower $\mid$ Def*|; see Fig. 12). This might be the result of the generally lower elasticity of system $\mathrm{B}$. At the shear rate of $0.5 \mathrm{~s}^{-1}$ in system A, for droplets larger than $92 \mu \mathrm{m}$ do not attain a steady shape, but the droplet eventually breaks up (see above); this size corresponds to a critical value of $\mathrm{Ca}$ for breakup of around 9, where the corresponding value of Def* ${ }_{c}$ is around -0.27 . In addition, the critical capillary number of system $\mathrm{A}$ is somewhat comparable to the steady state critical $\mathrm{Ca}$, which was around 6, in Lerdwijitjarud et al. (2002). Their system, PS(drop)/HDPE(matrix), like ours, had a viscosity ratio of unity and an $\mathrm{N}_{1}$ ratio of around 0.7. A droplet in system $\mathrm{B}$ breaks at a higher value of $\mathrm{Ca}(\sim 14)$ than for system A ( 9$)$. At the viscosity ratio of unity and $\mathrm{N}_{1}$ ratio of 2 , the steady state critical $\mathrm{Ca}$ was found to be around 12 in Lerdwijitjarud et al. (2002). This is slightly less than $\mathrm{Ca}_{\mathrm{c}}$ of system B. As shown in Fig. 2, the elasticity ratio $\left(\mathrm{G}_{\mathrm{r}}^{\prime}\right.$ at the selected shear rate) of system $\mathrm{B}$ is 3-4 times higher than that of system $\mathrm{A}$, which might account for the difference in critical capillary numbers for systems A and B. An alternative explanation might be that the weaker elasticity of system B produces less deformation in the vorticity direction than that of sys-

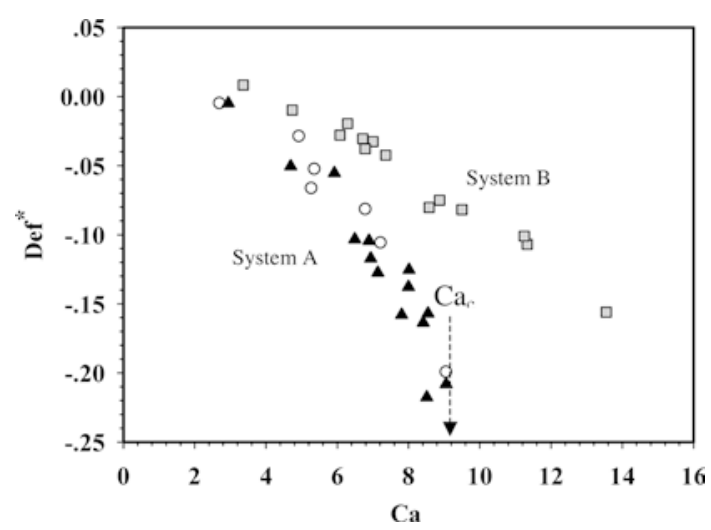

Fig. 12 Dependence of steady-state deformation parameter on $\mathrm{Ca}$ for system A: shear rate $0.3 \mathrm{~s}^{-1}$ (open circles), shear rate $0.5 \mathrm{~s}^{-1}$ (filled triangles), and for system B: shear rate $1 \mathrm{~s}^{-1}$ (shaded squares)] tem A at the same capillary number, and hence a greater shear rate is required to stretch droplets in system B to produce rupture. If this latter explanation is correct, then droplets with intermediate elasticity, high enough to avoid elongation in the flow direction, but low enough to avoid large elongations in the vorticity direction, will be most resistant to rupture and will break at the highest capillary number.

In our more elastic blend $\mathrm{A}$, when a constant shear rate above $\mathrm{Ca}_{\mathrm{c}}$ is applied, a spherical drop deforms nonmonotonically until regime five is reached and the droplet then elongates continuously in the vorticity direction until breakup occurs as shown in Fig. 10a-c. The droplet breaks when its two ends are quite far apart and no longer located on nearby streamlines, as shown in Fig. 13. Mighri and Huneault (2001) found that in a counter-rotating circular Couette cell, a viscoelastic droplet breaks when its two ends separated along the vorticity direction develop unstable motions because of large velocity differences between the two moving layers. A similar instability may occur in our flow.

We note that we could find no clear correlation in the literature or in our data between droplet Weissenberg number and re-orientation or break-up. The effect of elasticity may involve a complicated interplay of the first and second normal stress differences of both phases, and may couple to viscosity ratio, shear thinning, and capillary number. Careful experiments in which all these quantities are carefully controlled, along with numerical simulations of viscoelastic droplet deformation and

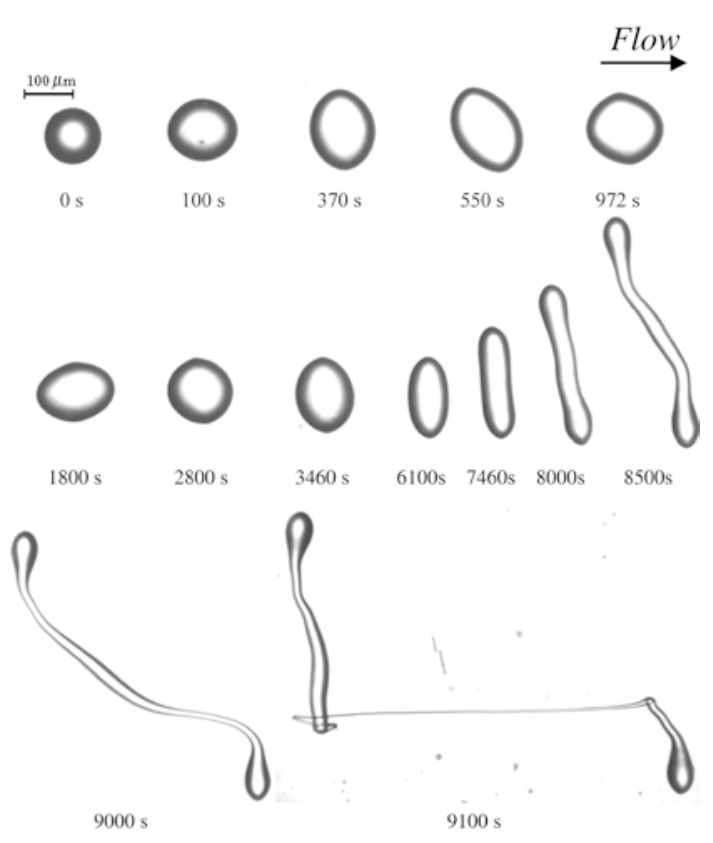

Fig. 13 Sequence of images during droplet breakup in system A, $\mathrm{D}_{\mathrm{o}}=120 \mu \mathrm{m}$, at a shear rate of $0.5 \mathrm{~s}^{-1}(\mathrm{Ca}=12)$. The flow direction is horizontal, and the vorticity direction vertical 
breakup will be needed to obtain a more quantitative picture of these fascinating phenomena.

\section{Conclusions}

We measured the dynamics of deformation of an elastic droplet in an elastic matrix by selecting two blend systems with viscosity ratio near unity, but of different elasticities of both droplet and matrix phases with the Weissenberg number of matrix phase around $0.1-0.5$ and of droplet phase around $0.2-0.5$. In start up of a steady shear flow, the different elasticities in the polymer blends produce qualitative differences in the droplet deformations that occur before the droplet attains its steady-state shape. In system A with higher elasticity, the deformation oscillates several times before reaching its steady-state shape. In system B with lower elasticity, the droplet first deforms in the shear direction, and thereafter continuously contacts in the flow direction until it reaches its steady-state shape. When the capillary number is increased at fixed shear rate (and hence fixed elasticity) by increasing the droplet size in system $\mathrm{A}$ and $\mathrm{B}$, the steady-state droplet shape becomes increasingly elongated in the vorticity direction and develops cusps along the vorticity axis. In this system $\mathrm{A}$, at still higher capillary number, droplet breakup occurs when two ends of a drop elongated in the vorticity direction are situated on streamlines of different velocity which pull the droplet ends apart, leading to rupture.

Acknowledgements The authors (W.L. and A.S.) would like to acknowledge the financial supports: the Thailand Research Fund (TRF), grants no. BRG/12/2544 and BRG4680015, the Petroleum and Petrochemical Technology Consortium grant.

\section{References}

Almusallam AS, Larson RG, Solomon MJ (2000) A constitutive model for the prediction of ellipsoidal droplet shapes and stresses in immiscible blends. J Rheol 44:1055-1083

Bartram E, Goldsmith HL, Mason SG (1975) Particle motions in non-Newtonian media. Rheol Acta 14:776-782

Bentley BJ, Leal LG (1986) An experimental investigation of drop deformation and breakup in steady, twodimensional linear flows. J Fluid Mech 167:241-283

Branrup J, Immergut EH (1989) Polymer handbook, 3rd edn, New York

Grace HP (1982) Dispersion phenomena in high viscosity immiscible fluid systems and application of static mixers as dispersion devices in such systems. Chem Eng Commun 14:225-227

Graebling D, Muller R, Palierne JF (1993) Linear viscoelastic behavior of some incompatible polymer blends in the melt. Interpretation of data with a model of emulsions of viscoelastic liquids. Macromolecules 26:320-329

Guido S, Villone M (1998) Three-dimensional shape of a drop under simple shear flow. J Rheol 42:395-415
Hobbie EK, Migler KB (1999) Vorticity elongation in polymeric emulsions. Phys Rev Lett 82:5393-5396

Larson RG (1999)The structure and rheology of complex fluids. Oxford University Press, New York

Lerdwijitjarud W, Sirivat A, Larson RG (2002) Influence of elasticity on dispersed-phase droplet size in immiscible polymer blends in simple shearing flow. Polym Eng Sci 42:798-809

Lerdwijitjarud W, Larson RG, Sirivat A, Solomon MJ (2003) Influence of weak elasticity of dispersed phase on droplet behavior in sheared polybutadiene/ poly(dimethylsiloxane) blends. J Rheol 47:37-57

Levitt L, Macosko CW, Pearson SD (1996) Influence of normal stress difference on polymer drop deformation. Polym Eng Sci 36:1647-1655

Luciani A, Champagne MF, Utracki LA (1997) Interfacial tension coefficient from the retraction of ellipsoidal drops. J Polym Sci Part B Polym Phys 35:13931403

Mighri F, Huneault MA (2001) Dispersion visualization of model fluids in a transparent Couette flow cell. J Rheol 45:783-797

Mighri F, Carreau PJ, Ajji A (1998) Influence of elastic properties on drop deformation and in shear flow. $\mathbf{J}$ Rheol 42:1477-1490
Migler KB (2000) Droplet vorticity alignment on model polymer blends. J Rheol 44:277-290

Palierne JF (1990) Linear rheology of viscoelastic emulsions with interfacial tension. Rheol Acta 29:204-214

Rumscheidt FD, Mason SG (1961) Particle motions in sheared suspensions. XII. Deformation and burst of fluid drops in shear and hyperbolic flow. J Coll Sci 16:238-261

Taylor GI (1932) The viscosity of a fluid containing small drops of another fluid. Proc R Soc A138:41-48

Taylor GI (1934)The formation of emulsions in definable fields of flow. Proc R Soc A146:501-523

Tsakalos VT, Navard P, Peurel-Disdier E (1998) Deformation and breakup mechanisms of single drops during shear. J Rheol 42:1403-1417

Wu S (1987) Formation of dispersed phase in incompatible polymer blends-interfacial and rheological effects. Polym Eng Sci 27:335-343

Yamane H, Takahashi M, Hayashi R, Okamoto K (1998) Observation of deformation and recovery of poly(isobutylene) droplet in a poly(isobutylene)/ poly(dimethyl siloxane) blend after application of step shear strain. J Rheol $42: 567-580$ 